\title{
EZ-structures and topological applications
}

\author{
F. T. Farrell* and J.-F. Lafont
}

\begin{abstract}
In this paper, we introduce the notion of an EZ-structure on a group, an equivariant version of the Z-structures introduced by Bestvina [4]. Examples of groups having an EZstructure include (1) torsion free $\delta$-hyperbolic groups, and (2) torsion free CAT( 0 )-groups.

Our first theorem shows that any group having an EZ-structure has an action by homeomorphisms on some $\left(\mathbb{D}^{n}, \Delta\right)$, where $n$ is sufficiently large, and $\Delta$ is a closed subset of $\partial \mathbb{D}^{n}=S^{n-1}$. The action has the property that it is proper and cocompact on $\mathbb{D}^{n}-\Delta$, and that if $K \subset \mathbb{D}^{n}-\Delta$ is compact, that diam $(g K)$ tends to zero as $g \rightarrow \infty$. We call this property $\left(*_{\Delta}\right)$.

Our second theorem uses techniques of Farrell-Hsiang [8] to show that the Novikov conjecture holds for any torsion-free discrete group satisfying condition $\left(*_{\Delta}\right)$ (giving a new proof that torsion-free $\delta$-hyperbolic and CAT(0) groups satisfy the Novikov conjecture).

Our third theorem gives another application of our main result. We show how, in the case of a torsion-free $\delta$-hyperbolic group $\Gamma$, we can obtain a lower bound for the homotopy groups $\pi_{n}(\mathcal{P}(B \Gamma))$, where $\mathcal{P}(\cdot)$ is the stable topological pseudo-isotopy functor.
\end{abstract}

\section{Introduction}

Let $\Gamma$ be a discrete group. Bestvina [4] defined the notion of a Z-structure on $\Gamma$ as a pair $(\bar{X}, Z)$ of spaces satisfying the following four axioms:

- $\bar{X}$ is a Euclidean retract (ER); i.e. it is locally contractible, contractible and has finite (covering) dimension.

- $Z$ is a Z-set in $\bar{X}$; i.e. $Z$ is a closed subset of $\bar{X}$ with the property that, for every open set $U \subset \bar{X}$, the inclusion $U-Z \hookrightarrow U$ is a homotopy equivalence.

- $\bar{X}-Z$ admits a free, properly discontinuous, cocompact action by the group $\Gamma$.

- The collection of translates of a compact set in $\bar{X}-Z$ forms a null sequence in $\bar{X}$; i.e. for every open cover $\mathcal{U}$ of $\bar{X}$, all but finitely many translates are $\mathcal{U}$ small.

Let us now introduce an equivariant version of a Z-structure:

Definition 1.1. We say that $(\bar{X}, Z)$ is an EZ-structure (equivariant Z-structure) on $\Gamma$ provided that $(\bar{X}, Z)$ is a $Z$-structure, and in addition, the $\Gamma$ action on $\bar{X}-Z$ extends to an action on $\bar{X}$.

\footnotetext{
*This research was supported in part by the National Science Foundation.
} 
Examples of groups with an EZ-structure include torsion-free $\delta$-hyperbolic groups [3] and CAT(0)-groups [4]. We note that a special case of a Z-structure on $\Gamma$ is the situation where $\bar{X}$ is a disk $\mathbb{D}^{n}$, and $Z=\partial \mathbb{D}^{n}=S^{n-1}$ :

Definition 1.2. We say that $\Gamma$ satisfies condition $(*)$ provided that there is an EZstructure of the form $\left(\mathbb{D}^{n}, S^{n-1}\right)$.

Farrell-Hsiang introduced this special case in [8] (see also [9], [12], [13]). Their motivation for the development of condition $(*)$ was that it provided an abstract setting under which the Novikov conjecture could be verified for the group $\Gamma$. Observe that there are groups with an EZ-structure that do not satisfy condition (*); for example, the free group on 2-generators. We now introduce a condition $\left(*_{\Delta}\right)$ for torsion-free groups, generalizing condition $(*)$. (For non torsion-free groups see Definition 3.1 below)

Definition 1.3. We say that $\Gamma$ satisfies condition $\left(*_{\Delta}\right)$ provided that there is an EZstructure of the form $\left(\mathbb{D}^{n}, \Delta\right)$, where $\Delta$ is a closed subset of $\partial \mathbb{D}^{n}=S^{n-1}$

We are now ready to state the first two theorems of this paper:

Theorem 1.1. Let $\Gamma$ be a discrete group, and assume that $\Gamma$ has an EZ-structure. Then $\Gamma$ satisfies condition $\left(*_{\Delta}\right)$.

Theorem 1.2. Let $\Gamma$ be a torsion-free discrete group satisfying condition $\left(*_{\Delta}\right)$. Then the Novikov conjecture holds for the group $\Gamma$.

The proofs of these theorems will be provided in Section 2 and Section 3 respectively. We note that the second theorem is not new, as Carlsson-Pederson [6] have already proven that groups with an EZ-structure satisfy this form of the Novikov conjecture. Nevertheless, the proof provided here is conceptually quite different from their argument (see Ferry-Weinberger [14] and $\mathrm{Hu}$ [16] for related results on the Novikov conjecture).

Now let us further restrict to groups which are torsion-free $\delta$-hyperbolic. For such a group $\Gamma$, Theorem 1.1 above ensures that the group satisfies condition $\left(*_{\Delta}\right)$. In fact, $\delta$-hyperbolicity ensures that the $\Gamma$-action on the pair $\left(\mathbb{D}^{n}, \Delta\right)$ has several additional properties. In Section 4, we will use these properties to show the following theorem:

Theorem 1.3. Let $\Gamma$ be a torsion-free $\delta$-hyperbolic group. Then for each integer $n \geq 0$, the group homomorphism:

$$
\bigoplus_{S \in \mathcal{M}} \pi_{n}\left(\phi_{S}\right): \bigoplus_{S \in \mathcal{M}} \pi_{n}(\mathcal{P}(B S)) \longrightarrow \pi_{n}(\mathcal{P}(B \Gamma))
$$

is monic. 
In the theorem above, $\mathcal{M}$ is a maximal collection of maximal infinite cyclic subgroups of $\Gamma$, with no two elements in $\mathcal{M}$ being conjugate, $\mathcal{P}(\cdot)$ is the stable topological pseudo-isotopy functor, and $\phi_{S}: \mathcal{P}(B S) \rightarrow \mathcal{P}(B \Gamma)$ is the functorially defined continuous map induced by $S \leq \Gamma$ (see Hatcher [15]). We refer the reader to Section 4 for a more complete discussion of this result.

Before starting with the proofs, we make a few comments concerning the results in this paper.

Remark 1. A natural question to ask is which finitely generated groups have an EZstructure? A version of this question was already posed by Bestvina [3], where he asks whether every group $\Gamma$ with a finite $B \Gamma$ has a Z-structure. It is interesting to construct groups which are neither $\delta$-hyperbolic, nor CAT( 0$)$ groups, but do have an EZ-structure. Bestvina gives some important examples of such groups in [3]. Do torsion free subgroups of finite index in $\mathrm{SL}_{n}(\mathbb{Z})$ have an EZ-structure?

Remark 2. It would also be of some interest to find applications of Theorem 1.1 to geometric group theory. Indeed, condition $\left(*_{\Delta}\right)$ for torsion free groups yields an action of the group on disks, which, aside from a "bad limit set" is properly discontinuous, fixed point free, and cocompact. With the exception of cocompactness, this is reminiscent of the action of a Kleinian group on (the compactification) of hyperbolic $n$-space. In some sense, Theorem 1.1 states that every torsion-free $\delta$ hyperbolic group has an action that mimics that of a Kleinian group. One feels that this should have some strong geometric consequences.

Remark 3. One could also consider the possibility of strengthening condition $\left(*_{\Delta}\right)$ by also requiring the action of the group $\Gamma$ on $\mathbb{D}^{n}$ to be smooth. Work of BenoistFoulon-Labourie [2] suggests that among $\delta$-hyperbolic groups, perhaps only uniform lattices satisfy this extra property. In any event it would be interesting to determine which $\delta$-hyperbolic groups satisfy this smooth form of condition $\left(*_{\Delta}\right)$.

\section{EZ-structure implies condition $\left(*_{\Delta}\right)$}

Let us fix a discrete group $\Gamma$ with an EZ-structure $(\bar{X}, Z)$. In this section we will provide a proof of Theorem 1.1. In order to do this, we will use the EZ-structure $(\bar{X}, Z)$ to build a new EZ-structure of the form $\left(\mathbb{D}^{n}, \Delta\right)$, where $\Delta$ is a closed subset of $\partial \mathbb{D}^{n}=S^{n-1}$. Let us start with a series of lemmas that will allow us to make the structure of $\bar{X}-Z$ more suitable to our purposes.

Lemma 2.1 (Reduction to a complex). Let $\Gamma$ be a group with an EZ-structure $(\bar{X}, Z)$. Then there is an EZ-structure $(\tilde{K} \cup Z, Z)$, where $\tilde{K}$ is the universal cover of a finite simplicial complex. 
Proof. We first observe that the hypotheses for an EZ-structure imply that the group $\Gamma$ is the fundamental group of an aspherical compact ANR, namely $(\bar{X}-Z) / \Gamma$. By a result of West [24], any compact ANR is homotopy equivalent to a compact polyhedra $K$. In particular $K$ is a $K(\Gamma, 1)$. A result of Bestvina (Lemma 1.4 in [4]) now implies that $(\tilde{K} \cup Z, Z)$ is an EZ-structure.

Our next step is to "fatten" $K$ so that it is a manifold with boundary. In order to do this, we embed (simplicially) $K$ into a high dimensional ( $n \geq 5$ ) copy of $\mathbb{R}^{n}$, and let $W$ be a regular neighborhood of $K$. Note that $W$ is a compact manifold with boundary, and denote by $r: W \rightarrow K$ a retraction of $W$ onto $K$. Let the retraction $\tilde{r}: \tilde{W} \rightarrow \tilde{K}$ be the $\Gamma$-equivariant lift of $r$.

Lemma 2.2 (Reduction to a manifold with boundary). The pair $(\tilde{W} \cup Z, Z)$ is an EZ-structure for $\Gamma$.

Proof. We follow the argument of Lemma 1.4 in Bestvina [4]. We start by taking the diagonal embedding of $\tilde{W}$ in $(\tilde{W} \cup \infty) \times(\tilde{K} \cup Z)$. The first factor is the one point compactification of $\tilde{W}$, while the map into the second factor is given by $\tilde{r}: \tilde{W} \rightarrow$ $\tilde{K} \hookrightarrow \tilde{K} \cup Z$. The topology on $\tilde{K} \cup Z$ comes from taking the closure of the image of this diagonal embedding. Lemma 1.3 in Bestvina [4] shows that this is a Z-structure. Furthermore, by construction, the action of $\Gamma$ on $\tilde{W}$ extends to an action of $\Gamma$ on $\tilde{W} \cup Z$. Hence we have an EZ-structure.

An identical argument can be used to show the following:

Lemma 2.3 (Doubling across the boundary). Let $(N \cup Z, Z)$ be an EZ-structure on $\Gamma$, and assume that $N$ is a manifold (with or without boundary). Denote by $\mathcal{N}$ the space $(N \times I) / \equiv$, where we collapse each $p \times I, p \in \partial N$, to a point (so if $N$ has no boundary, then $\mathcal{N}=N \times I)$. Then $(\mathcal{N} \cup Z, Z)$ is an EZ-structure on $\Gamma$.

Proof. We proceed as in the previous lemma, using the obvious $\Gamma$-equivariant map $\rho: \mathcal{N} \rightarrow N \hookrightarrow N \cup Z$ in the place of $\tilde{r}$. That is to say, we embed $\mathcal{N}$ into the space $(\mathcal{N} \cup \infty) \times(N \cup Z)$ using the inclusion map on the first factor, and the map $\rho$ on the second factor. $\mathcal{N} \cup Z$ is then the closure of the image of $\mathcal{N}$ under this map, with the induced topology. Once again, $Z$ lies as a $Z$-set, and the mapping is $\Gamma$-equivariant by construction.

Note that the space $\mathcal{N}$ defined in Lemma 2.3 is also a manifold with boundary, and that the boundary $\partial \mathcal{N}$ of $\mathcal{N}$ is by construction just the double of $N$ (the two copies being $N \times\{0\}$ and $N \times\{1\}$ ).

We now return to the situation we are interested in. We have shown that we can reduce to the case where the EZ-structure is of the form $(\tilde{W} \cup Z, Z)$, where $\tilde{W}$ is a manifold with boundary. This allows us to apply the construction from the previous 
lemma to obtain a new EZ-structure $(\mathcal{W} \cup Z, Z)$. Our next result shows that $\mathcal{W} \cup Z$ is in fact a topological manifold. Because we will be referring to this result later in this section, we prove it in a slightly more general form.

Proposition 2.1. Let $(N \cup Z, Z)$ be an EZ-structure on $\Gamma$, and assume that $N$ is a manifold (with or without boundary) of dimension $\geq 5$. Let $(\mathcal{N} \cup Z, Z)$ be the EZstructure defined in Lemma 2.3. Then the space $\mathcal{N} \cup Z$ is a manifold with boundary.

Proof. In order to show that the space $\mathcal{N} \cup Z$ is a compact manifold with we will use the celebrated characterization of high dimensional topological manifolds due to Edwards and Quinn (for a pleasant general survey, we refer to Mio [20]). Recall that this characterization provides a list of five necessary and sufficient conditions for a locally compact high dimensional topological space to be a closed topological manifold. The corresponding characterization for manifolds with boundary requires an additional condition about the 'boundary'. We will verify each of these six conditions as a separate claim.

Claim 1 (Finite dimensional). The space $\mathcal{N} \cup Z$ is finite dimensional.

Claim 2 (Locally contractible). The space $\mathcal{N} \cup Z$ is locally contractible.

Proof. These follow from the fact that the pair $(\mathcal{N} \cup Z, Z)$ is a Z-structure. Indeed, the first condition for a Z-structure forces $\mathcal{N} \cup Z$ to be an ER, and ER's are locally contractible and finite dimensional.

Claim 3 (Homology manifold). The space $\mathcal{N} \cup Z$ is a homology manifold with boundary.

Proof. Let $n$ be the dimension of the manifold $N$. We need to verify that the local homology of every point is either that of an $(n+1)$-dimensional sphere (for "interior" points) or that of a point (for "boundary" points). In order to do this, we first observe that the local homology is easy to compute for points in $\mathcal{N}$. Indeed, $\mathcal{N}$ is actually a manifold with boundary, hence the local homology has the correct values.

Now let us focus on a point $p$ that lies on $Z \subset \mathcal{N} \cup Z$. We claim that the (reduced) local homology at $p$ is trivial. So we need to show that $\bar{H}_{*}((\mathcal{N} \cup Z),(\mathcal{N} \cup Z)-p)=0$. But this is also an immediate consequence of the fact that $Z$ is a Z-set in $\mathcal{N} \cup Z$. Indeed, an equivalent formulation of the Z-set property states that there is a homotopy $J:(\mathcal{N} \cup Z) \times I \rightarrow \mathcal{N} \cup Z$ which satisfies the conditions:

- $J$ maps $\mathcal{N} \times I$ into $\mathcal{N}$.

- $J_{0}:(\mathcal{N} \cup Z) \times\{0\} \rightarrow \mathcal{N} \cup Z$ is the identity map.

- $J_{t}:(\mathcal{N} \cup Z) \times\{t\} \rightarrow \mathcal{N} \cup Z$ maps into $\mathcal{N}$ for all $t>0$. 
In particular, the homotopy $J$ gives a family of homotopic maps which respect the pair $((\mathcal{N} \cup Z),(\mathcal{N} \cup Z)-p)$, hence they all induce the same maps on the level of the homology groups $\bar{H}_{*}((\mathcal{N} \cup Z),(\mathcal{N} \cup Z)-p)$. But the map induced by $J_{0}$ is the identity map, while the map induced by $J_{1}$ is the trivial map (since $\left.J_{1}(\mathcal{N} \cup Z) \subset \mathcal{N} \subset(\mathcal{N} \cup Z)-p\right)$. Hence we have that the identity map coincides with the zero map, which immediately implies that $\bar{H}_{*}((\mathcal{N} \cup Z),(\mathcal{N} \cup Z)-p)$ is trivial. We conclude that $\mathcal{N} \cup Z$ is indeed a homology manifold with boundary.

Let us now recall the definition of the disjoint disk property. A topological space $X$ has the disjoint disk property provided that any pair of maps from $\mathbb{D}^{2}$ into a space $X$ can be approximated, to an arbitrary degree of precision, by maps whose images are disjoint.

Claim 4 (Disjoint disk property). The space $\mathcal{N} \cup Z$ has the disjoint disk property.

Proof. Note that, since $\mathcal{N} \cup Z$ is an ER, it is metrizable; we will use this metric to measure the closeness of maps. Let $f, g$ be arbitrary maps from $\mathbb{D}^{2}$ into $\mathcal{N} \cup Z$, and let $\epsilon>0$ an arbitrary real number. We need to exhibit a pair of maps which are $\epsilon$ close to the maps we started with, and have disjoint image.

Observe that, since $Z$ is a $Z$-set in the space $\mathcal{N} \cup Z$, there is a map $H: \mathcal{N} \cup Z \rightarrow \mathcal{N}$ with the property that $H$ is an $(\epsilon / 2)$-approximation of the identity map on $\mathcal{N} \cup Z$. Consider the compositions $f^{\prime}:=H \circ f$ and $g^{\prime}:=H \circ g$, and observe that the maps $f^{\prime}$ and $g^{\prime}$ are $(\epsilon / 2)$-approximations of $f$ and $g$ respectively. Furthermore, $f^{\prime}$ and $g^{\prime}$ map $\mathbb{D}^{2}$ into the subset $\mathcal{N}$, which we know is a manifold of dimension $\geq 6$.

But high dimensional manifolds automatically have the disjoint disk property, so we can find $(\epsilon / 2)$-approximations $f^{\prime \prime}, g^{\prime \prime}$ to the maps $f^{\prime}, g^{\prime}$ whose images are disjoint. It is immediate from the triangle inequality that the $f^{\prime \prime}, g^{\prime \prime}$ satisfy our desired properties. Hence the space $\mathcal{N} \cup Z$ has the disjoint disk property.

Claim 5 (Manifold point). The space $\mathcal{N} \cup Z$ has a manifold point.

Proof. By a manifold point, we mean a point with a neighborhood homeomorphic to some $\mathbb{R}^{n+1}$. This is clear, since $\mathcal{N}$ is actually a topological $(n+1)$-dimensional manifold.

We now remind the reader of the characterization of high dimensional topological manifolds due to Edwards-Quinn ([7], [22], [23]):

Theorem 2.1 (Characterization of topological manifolds). Let $X$ be a locally compact topological space, $n \geq 5$ an integer. Assume that $X$ satisfies the following properties:

- X has the local homology of an n-dimensional manifold.

- $X$ is locally contractible. 
- X has finite (covering) dimension.

- $X$ satisfies the disjoint disk property.

Then there is a locally defined invariant $I(X) \in 8 \mathbb{Z}+1$ with the property that $X$ is a topological manifold if and only if $I(X)=1$.

The corresponding theorem for a manifold with boundary requires an additional modification of the first condition. Namely, one needs to replace it with the following:

- every point $p \in X$ has either the local homology of an $n$-dimensional sphere, or that of a point.

- the subset of points having the local homology of a point, denoted by $\partial_{h}(X)$ (the "homological" boundary), is a topological manifold of dimension $n-1$.

Under these two conditions, the Edwards-Quinn result implies that the space $X$ is a topological manifold with boundary (and the set $\partial_{h}(X)$ is the boundary of the manifold $X$ ) if and only if the locally defined invariant $I(X)=1$ (see Theorem 3.4.2 in Quinn [21]).

As such, we have reduced our theorem to showing the following:

Claim 6. The set $\partial_{h}(\mathcal{N} \cup Z)$ is a compact manifold of dimension one lower than the dimension of $\mathcal{N}$.

Proof. By the proof of claim 3, we know exactly what the set $\partial_{h}(\mathcal{N} \cup Z)$ is. Namely, it consists of the set $\partial \mathcal{N} \cup Z$. Note that the set $\partial \mathcal{N}$ is just the double of $N$ across it's boundary. In particular, $\partial_{h}(\mathcal{N} \cup Z)$ is obtained by taking two copies of $N \cup Z$, and identifying the two copies of $\partial N \cup Z$.

We now claim that $\partial N \cup Z$ is a Z-set in the space $N \cup Z$. In order to show this we need to exhibit a map $f_{\epsilon}: N \cup Z \rightarrow N \cup Z$ that is $\epsilon$-close to the identity, and has $f_{\epsilon}(N \cup Z) \subset N-\partial N$. Note that since $Z$ is a Z-set in $N \cup Z$, there is a map $g$ that is $(\epsilon / 2)$-close to the identity, and maps $N \cup Z$ into $N$. Next, observe that since $N$ itself is a manifold with boundary, $\partial N$ is a Z-set in $N$, which implies the existence of a map $h: N \rightarrow N-\partial N$ which is $(\epsilon / 2)$-close to the identity. Composing the two maps and using the triangle inequality gives us our desired claim.

So we see that $\partial_{h}(\mathcal{N} \cup Z)$ is obtained by doubling a Z-compactification $N \cup Z$ of an open manifold $\operatorname{Int}(N)$ along it's Z-boundary $\partial N \cup Z$. By a result of AncelGuilbault (Theorem 9 in [1]), this is automatically a manifold. The dimension claim comes from the fact that $\partial_{h}(\mathcal{N} \cup Z)$ contains $\partial \mathcal{N}$, hence must be a manifold of the same dimension as $\partial \mathcal{N}$, which is one less than the dimension of $\mathcal{N}$.

The Edwards-Quinn result now applies, completing our proof of Proposition 2.1.

Let us summarize what we have so far: if $\Gamma$ has an EZ-structure, we have shown that there is an EZ-structure ( $\mathcal{W} \cup Z, Z$ ) with the additional property that $\mathcal{W} \cup Z$ is a topological manifold, and $Z$ is a closed subset in the boundary of the topological 
manifold. We now want to further improve the EZ-structure so that the space is in fact a topological disk. In order to do this, we iterate our procedure once more and define the space $\mathbb{W}=(\mathcal{W} \times I) / \equiv$, where again the equivalence relation is given by collapsing $p \times I, p \in \partial \mathcal{W}$ to a point. By Lemma 2.3, the pair ( $\mathbb{W} \cup Z, Z$ ) is again an EZ-structure for $\Gamma$, and by Proposition 2.1, $\mathbb{W} \cup Z$ is a topological manifold with boundary. We claim that $\mathbb{W} \cup Z$ is in fact a topological disk.

Proposition 2.2. The space $\mathbb{W} \cup Z$ is a disk.

Proof. We begin by showing that the space $\partial(\mathbb{W} \cup Z)$ is simply connected. Notice that $\partial(\mathbb{W} \cup Z$ ) is the double of the compact manifold with boundary $W \cup Z$ along its boundary $\partial \mathcal{W} \cup Z$. Furthermore each of the spaces $\mathcal{W} \cup Z$ is contractible. Seifert-Van Kampen now yields that the double $\partial(\mathbb{W} \cup Z$ ) must be simply connected. Furthermore, observe that the space $\mathbb{W} \cup Z$ is contractible.

Finally we note that any compact contractible manifold of dimension $\geq 6$ with simply connected boundary must be homeomorphic to a disk. This is a well known consequence of the h-cobordism theorem. A proof in the smooth category can be found in Chapter 9, Proposition A, of Milnor's book [19]. The same proof holds verbatim, replacing the use of Smale's smooth h-cobordism theorem with the topological h-cobordism theorem of Kirby-Siebenmann's [18]. This concludes our proof of the proposition.

We have shown how given an arbitrary EZ-structure on a discrete group $\Gamma$, we can construct an EZ-structure of the form $\left(\mathbb{D}^{n}, \Delta\right)$, where $\Delta$ is a closed subset of $\partial \mathbb{D}^{n}=S^{n-1}$. In particular, we see that any group which has an EZ-structure automatically satisfies condition $\left(*_{\Delta}\right)$.

\section{Condition $\left(*_{\Delta}\right)$ implies the Novikov conjecture}

We start this section by giving a reformulation of condition $\left(*_{\Delta}\right)$ which is closer to the formulation given by Farrell-Hsiang:

Definition 3.1. We say that a group $\Gamma$ satisfies condition $\left(*_{\Delta}\right)$ if for some integer $n$ there is an action of $\Gamma$ on $\left(\mathbb{D}^{n}, \Delta\right), \Delta$ a closed subset of $S^{n-1}=\partial \mathbb{D}^{n}$ with the following two properties:

- $\Gamma$ acts properly discontinuously and cocompactly on $\mathbb{D}^{n}-\Delta$,

- for each compact subset $K$ of $\mathbb{D}^{n}-\Delta$, and each $\epsilon>0$, there exists a $\delta=$ $\delta(K, \epsilon)>0$ such that for each $\gamma \in \Gamma$, if $d(\gamma K, \Delta)<\delta$, then $\operatorname{diam}(\gamma K)<\epsilon$.

Observe that condition $\left(*_{\Delta}\right)$ generalizes condition $(*)$ formulated in FarrellHsiang [8] (the reader is also referred to [9] and the survey papers [12], [13]). The 
only difference between the two conditions is that condition $(*)$ also required the set $\Delta$ to be $\partial \mathbb{D}^{n}=S^{n-1}$, and $\Gamma$ to be torsion-free. Furthermore, for torsion-free groups, it is easy to see that condition $\left(*_{\Delta}\right)$ corresponds exactly to the existence of an EZ-structure of the form $\left(\mathbb{D}^{n}, \Delta\right)$, where $\Delta$ is a closed subset of $S^{n-1}$.

Note that, by the theorem proved in the previous section, any group which has an EZ-structure automatically satisfies condition $\left(*_{\Delta}\right)$. In particular, the following two families of groups satisfy condition $\left(*_{\Delta}\right)$ :

- torsion-free $\delta$-hyperbolic groups.

- torsion-free CAT(0)-groups.

Before starting the proof of Theorem 1.2, we first state the following useful lemma:

Lemma 3.1. Let $\left(\mathbb{D}^{m}, \Delta\right)$ be a $\Gamma$-space satisfying the properties given in condition $\left(*_{\Delta}\right)$. Then there is a second $\Gamma$-space $\left(\mathbb{D}^{m+1}, \Delta\right)$ also satisfying $\left(*_{\Delta}\right)$, and a continuous $\Gamma$-equivariant surjection $\mathbb{D}^{m} \times I \rightarrow \mathbb{D}^{m+1}$ mapping $\Delta \times I$ to $\Delta$ and mapping $\left(\mathbb{D}^{m}-\Delta\right) \times I$ homeomorphically to $\mathbb{D}^{m+1}-\Delta$.

Proof. Let $\bar{X}=\left(\mathbb{D}^{m} \times I\right) / \equiv$, where the equivalence relation collapses each line segment $x \times I, x \in \Delta$, to a point. Let $\phi: \mathbb{D}^{m} \times I \rightarrow \bar{X}$ be the quotient map, and give $\bar{X}$ the $\Gamma$-space structure such that $\phi$ is $\Gamma$-equivariant. Clearly, $\left.\phi\right|_{\left(\mathbb{D}^{m}-\Delta\right) \times I}$ is a homeomorphism onto $\bar{X}-\Delta$.

Projection onto the first factor of $\mathbb{D}^{m} \times I$ induces a $\Gamma$-equivariant map $\Psi: \bar{X}-\Delta \rightarrow \mathbb{D}^{m}$. The topology on $\bar{X}=(\bar{X}-\Delta) \cup \Delta$ induced, using $\Psi$, by the construction in Lemma 2.2 coincides with the one described above, as both topologies are compact and Hausdorff. Hence $(\bar{X}, \Delta)$ is an EZ-structure on $\Gamma$.

It remains to show that $\bar{X}$ is homeomorphic to $\mathbb{D}^{m+1}$. For this we introduce a second decomposition space $Y=\mathbb{D}^{m} \times[0,2] / \sim$, where $\sim$ collapses each line segment $x \times[0,1], x \in \Delta$, to a point. Since $Y$ and $\bar{X}$ are clearly homeomorphic, it suffices to construct a homeomorphism from $Y$ to $\mathbb{D}^{m} \times[0,2]$. To do this, let $\phi: \mathbb{D}^{m} \rightarrow[0,1]$ be a continuous function such that $\phi^{-1}(0)=\Delta$. Define $f: \mathbb{D}^{m} \times[0,2] \rightarrow \mathbb{D}^{m} \times[0,2]$ to be $f(x, t)=(x, t \phi(x))$ if $0 \leq t \leq 1$, and $f(x, t)=(x,(2-\phi(x)) t+2 \phi(x)-2)$ if $1 \leq t \leq 2$. Observe that $f$ is a surjection.

Since the point inverses of $f$ give the decomposition $\sim$ of $\mathbb{D}^{m} \times[0,2], f$ induces the desired homeomorphism.

The condition $(*)$ was introduced by Farrell-Hsiang in order to provide an abstract setting in which Novikov's Conjecture could be verified. But the proof given in their paper carries over almost verbatim to the more general setting of condition $\left(*_{\Delta}\right)$. Namely the following is true:

Theorem 3.1. Let $\left(\mathbb{D}^{m}, \Delta\right)$ be a $\Gamma$-space with the properties given in condition $\left(*_{\Delta}\right)$. Suppose that $\Gamma$ is torsion-free, and let $M^{m}$ denote the orbit space $\left(\mathbb{D}^{m}-\Delta\right) / \Gamma$. 
Observe that $M^{m}$ is an aspherical compact manifold with boundary. Then the map in the (simple) surgery exact sequence:

$$
\varsigma^{s}\left(M^{m} \times \mathbb{D}^{n}, \partial\right) \longrightarrow\left[M^{m} \times \mathbb{D}^{n}, \partial ; G / \text { Top }\right]
$$

is identically zero when $n \geq 1$ and $n+m \geq 6$.

Proof. For the reader's convenience, we recall the argument of [8] for the special case where $\Gamma$ satisfies condition $(*)$, as exposited in the Trieste notes [13], emphasizing the modifications needed for the more general setting of condition $\left(*_{\Delta}\right)$. So as not to obscure the argument, we assume that $n=1$ and $M^{m}$ is triangulable. Notice that the Lemma 3.1 formally reduces the general case $n \geq 1$ to the special case $n=1$.

Let $\left(\mathbb{D}^{m+1}, \Delta\right)$ be the $\Gamma$-space determined by applying Lemma 3.1 to the $\Gamma$-space $\left(\mathbb{D}^{m}, \Delta\right)$, and notice that $M^{m} \times \mathbb{D}^{1}=\left(\mathbb{D}^{m+1}-\Delta\right) / \Gamma$. Define the space:

$$
\varepsilon^{2 m+1}=\left(\mathbb{D}^{m+1}-\Delta\right) \times_{\Gamma}\left(\mathbb{D}^{m}-S^{m-1}\right)
$$

and let $p: \varepsilon^{2 m+1} \rightarrow M^{m} \times \mathbb{D}^{1}$ be the bundle projection induced by the projection to the first factor (the fiber of this projection is $\mathbb{D}^{m}-S^{m-1}$ ). Then the following diagram commutes:

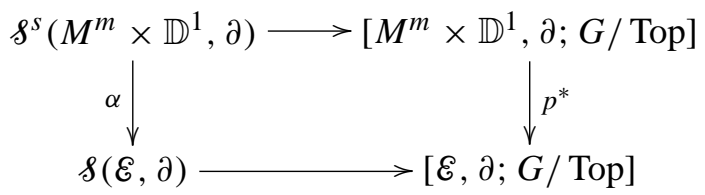

where $\alpha$ is the obviously defined transfer map (see [13], pgs. 246-247). Since $p$ is a homotopy equivalence, $p^{*}$ is an isomorphism. Hence to prove the theorem, it is sufficient to verify the following:

Assertion. The map $\alpha$ is identically zero.

To verify this assertion, note first that an arbitrary element in $\varsigma^{s}\left(M^{m} \times \mathbb{D}^{1}, \partial\right)$ can be represented by a pair $(f, h)$, where $f: M^{m} \rightarrow M^{m}$ is a self-homeomorphism with $\left.f\right|_{\partial M^{m}}=\operatorname{Id}_{\partial M^{m}}$, and $h: M^{m} \times \mathbb{D}^{1} \rightarrow M^{m} \times \mathbb{D}^{1}$ is a homotopy of $f$ to $\operatorname{Id}_{M^{m}}$ relative $\partial M^{m}$. Define:

$$
E^{2 m}=\left(\mathbb{D}^{m}-\Delta\right) \times_{\Gamma}\left(\mathbb{D}^{m}-S^{m-1}\right)
$$

and notice that by Lemma 3.1, we have that $\varepsilon^{2 m+1}=E^{2 m} \times I$.

Observe that, given such a pair $(f, h)$, there is a well defined lift $\tilde{f}: \mathbb{D}^{m}-\Delta \rightarrow$ $\mathbb{D}^{m}-\Delta$, and that $\left.\tilde{f}\right|_{S^{m-1}-\Delta}=\operatorname{Id}_{S^{m-1}-\Delta}$. Now let $\tilde{h}$ be the unique lift of $h$ to $\left(\mathbb{D}^{m}-\Delta\right) \times I=\mathbb{D}^{m+1}-\Delta$ with the property that $\tilde{h}$ is a proper homotopy equivalence (relative $S^{m-1}-\Delta$ ) between $\operatorname{Id}_{\mathbb{D}^{m}-\Delta}$ and the self-homeomorphism $\tilde{f}$. 
Then $k:=\tilde{h} \times \mathrm{Id}_{\mathbb{D}^{m}-S^{m-1}}$ determines a proper homotopy (relative $\partial E$ ):

$$
k: \mathcal{E}=E \times I \longrightarrow E \times I
$$

between $\operatorname{Id}_{E}$ and a self-homeomorphism $g: E \rightarrow E$ (which is also determined by $\left.\tilde{f} \times \operatorname{Id}_{\mathbb{D}^{m}-S^{m-1}}\right)$. Note that $\delta(\mathcal{E}, \partial)=\delta(E \times I, \partial)$, since $\mathcal{E}=E \times I$. Hence the pair $(g, k)$ represents the image of the pair $(f, h)$ under the transfer map, i.e. $(g, k)=$ $\alpha(f, h)$. The assertion then claims that the pair $(g, k)$ obtained in this manner is always zero in $\delta(\mathcal{E}, \partial)$. In particular, the assertion would follow from the following:

Proposition 3.1. $g$ is pseudo-isotopic to $\operatorname{Id}_{E}$ (relative $\partial E$ ), via a pseudo-isotopy which is properly homotopic to $k$ (relative $\partial$ ).

We will now use the condition $\left(*_{\Delta}\right)$ to construct the pseudo-isotopy posited in this proposition. Start by defining a new space $\bar{E}:=\mathbb{D}^{m} \times_{\Gamma}\left(\mathbb{D}^{m}-S^{m-1}\right)$. Note that the projection onto the second factor determines a fiber bundle projection $q: \bar{E} \rightarrow$ $\operatorname{Int}\left(M^{m}\right)$ with fiber $\mathbb{D}^{m}$ (recall that $\left.\operatorname{Int}\left(M^{m}\right)=\left(\mathbb{D}^{m}-S^{m-1}\right) / \Gamma\right)$. Hence $\bar{E}$ is a manifold containing $E$ as an open dense subset, and $\partial E \subset \partial \bar{E}$.

Next observe that the second property of condition $\left(*_{\Delta}\right)$ implies that $\tilde{f}$ extends to a $\Gamma$-equivariant homeomorphism $\bar{f}: \mathbb{D}^{m} \rightarrow \mathbb{D}^{m}$ by setting $\left.\bar{f}\right|_{S^{m-1}}=\operatorname{Id}_{S^{m-1}}$. Consequently, $\bar{f} \times \operatorname{Id}_{\mathbb{P}^{m}-\Delta}$ determines a self-homeomorphism $\bar{g}: \bar{E} \rightarrow \bar{E}$ which extends $g: E \rightarrow E$ and satisfies $\left.\bar{g}\right|_{\partial \bar{E}}=\operatorname{Id}_{\partial \bar{E}}$. We now proceed to construct a pseudo-isotopy $\phi: \bar{E} \times I \rightarrow \bar{E} \times I$ satisfying:

- $\left.\phi\right|_{\bar{E} \times\{0\}}=\bar{g}$

- $\left.\phi\right|_{\bar{E} \times\{1\}}=\operatorname{Id}_{\bar{E} \times\{1\}}$

- $\left.\phi\right|_{(\partial \bar{E}) \times I}=\operatorname{Id}_{(\partial \bar{E}) \times I}$.

Once this is done, then the restriction of $\phi$ to the subset $E \times I \subset \bar{E} \times I$ will be the pseudo-isotopy posited in the proposition.

Observe that the three properties stated above define $\phi$ on the entire set $\partial(\bar{E} \times I)$. We need to extend $\phi$ over $\operatorname{Int}(\bar{E} \times I)$. In order to do this, consider the fiber bundle $r: \bar{E} \times I \rightarrow \operatorname{Int}(M)$ with fiber $\mathbb{D}^{m} \times I$, where $r$ is the composition of the projection onto the first factor of $\bar{E} \times I$ followed by the map $q: \bar{E} \rightarrow \operatorname{Int}(M)$. Observe that if $\sigma$ is an $n$-simplex in a triangulation of $\operatorname{Int}(M)$, then $r^{-1}(\sigma)$ can be identified with $\mathbb{D}^{n+m+1}$.

The construction of $\phi$ proceeds by induction over the skeleta of $\operatorname{Int}(M)$ via a standard obstruction theory argument. And the obstructions encountered in extending $\phi$ from the $(n-1)$-skeleton to the $n$-skeleton are precisely those of extending a self-homeomorphism of $S^{n+m}$ to a self-homeomorphism of $\mathbb{D}^{n+m+1}$. But these obstructions all vanish, because of the Alexander Trick. Recall that this trick asserts that any self-homeomorphism $\eta$ of $S^{n}$ extends to a self-homeomorphism $\bar{\eta}$ of $\mathbb{D}^{n+1}$. In fact, $\bar{\eta}(t x)=t \eta(x)$ where $x \in S^{n}$ and $t \in I$ is an explicit extension. 
Now the restriction $\psi:=\left.\phi\right|_{E \times I}$ is the pseudo-isotopy from $g$ to $\operatorname{Id}_{E}$ asserted in the proposition. A similar argument, which we omit, shows that $\psi$ is properly homotopic to $k$ relative $\partial$. This concludes the proof.

\section{Bounding $\pi_{n}(\mathcal{P}(B \Gamma))$ for $\delta$-hyperbolic groups}

In this section, we give an application of our main result to obtaining a lower bound for the homotopy groups $\pi_{n}(\mathscr{P}(B \Gamma))$ which holds for all torsion-free $\delta$-hyperbolic groups $\Gamma$. Here $\mathcal{P}(\cdot)$ is the stable topological pseudo-isotopy functor (see Hatcher [15]). For this we need to first recall some basic facts about $\delta$-hyperbolic groups. Let $\Gamma$ be a torsion free $\delta$-hyperbolic group (we exclude the case $\Gamma=\mathbb{Z}$ ). Then the following are true:

Fact 1. If $S$ is an infinite cyclic subgroup of $\Gamma$, then there is a maximal infinite cyclic subgroup containing $S$. Furthermore this maximal subgroup is unique.

Fact 2. If $C$ is a maximal infinite cyclic subgroup of $\Gamma$, then its normalizer is $C$ itself.

Fact 3. If $S_{1}$ and $S_{2}$ are a pair of maximal infinite cyclic subgroups of $\Gamma$, and $\left\{S_{i}^{ \pm}\right\} \subset \partial^{\infty} \Gamma$ are the corresponding pair of points in the boundary at infinity, then either $S_{1}=S_{2}$ or $\left\{S_{1}^{ \pm}\right\} \cap\left\{S_{2}^{ \pm}\right\}=\emptyset$.

Fact 4. If $S$ is a maximal infinite cyclic subgroup of $\Gamma$, then $\gamma \cdot S^{-} \neq S^{+}$for all $\gamma \in \Gamma$.

We briefly explain why each of these facts holds. The existence part of Fact 1 follows from Proposition 3.16 in Bridson-Haefliger (pg. 465 in [5]), while uniqueness follows from Fact 3 . For a maximal infinite cyclic subgroup, the normalizer coincides with the centralizer. If the element is not in the group itself, this would yield a pair of commuting elements, giving a $\mathbb{Z}^{2}$ in $\Gamma$, which is impossible, giving us Fact 2 . Fact 3 follows from the proof of Theorem 3.20 in Bridson-Haefliger (pg. 467 in [5]). Fact 4 is an easy consequence of Facts 2 and 3.

Now fix a set $\mathcal{M}$ where the elements of $\mathcal{M}$ are maximal infinite cyclic subgroups of $\Gamma$ with each conjugacy class represented exactly once. For each $S \in \mathcal{M}$, let $\phi_{S}: \mathcal{P}(B S) \rightarrow \mathcal{P}(B \Gamma)$ be the functorially defined continuous map (see Hatcher [15]). Note that $B S=S^{1}$ for each $S \in \mathcal{M}$. Theorem 1.3 that we are going to prove in this section states that, for each integer $n \geq 0$, the group homomorphism

$$
\bigoplus_{S \in \mathcal{M}} \pi_{n}\left(\phi_{S}\right): \bigoplus_{S \in \mathcal{M}} \pi_{n}(\mathcal{P}(B S)) \longrightarrow \pi_{n}(\mathcal{P}(B \Gamma))
$$

is an injection. 
Note that $\pi_{0}\left(\mathcal{P}\left(S^{1}\right)\right) \cong \mathbb{Z}_{2} \oplus \mathbb{Z}_{2} \oplus \cdots$, where there are countably infinite number of $\mathbb{Z}_{2}$ 's (see Igusa [17]). Furthermore, the Isomorphism Conjecture for $\mathcal{P}(B \Gamma)$ formulated by Farrell-Jones [11] is equivalent to the assertion that the homeomorphisms in Theorem 1.3 are all isomorphisms together with the assertion that the Whitehead groups $W h\left(\Gamma \times \mathbb{Z}^{n}\right)$ vanish for all $n$.

Let us now proceed to prove Theorem 1.3. By Theorem 1.1, we know that we have a sequence of EZ-structures $\left(\mathbb{D}^{m}, \partial^{\infty} \Gamma\right)$, defined for all sufficiently large $m$, such that $\Gamma$ acts on $\mathbb{D}^{m}$ by orientation preserving homeomorphisms, and $\left(\mathbb{D}^{m+1}, \partial^{\infty} \Gamma\right)=$ $\left(\mathbb{D}^{m}, \partial^{\infty} \Gamma\right) \times I$ (i.e. is $\mathbb{D}^{m} \times I / \equiv$ where each interval $x \times I$, with $x \in S^{m-1}$, is collapsed to a point). Furthermore, each $S \in \mathcal{M}$ determines a pair of distinct points $S^{+}, S^{-} \in \partial^{\infty} \Gamma$. We start our argument by showing:

Claim 1. $\left(\mathbb{D}^{m},\left\{S^{ \pm}\right\}\right)$is an EZ-structure for $S$.

Proof. To see this claim, we first note that a closed subset of a Z-set is still a Z-set, hence the pair $\left(\mathbb{D}^{m}, S^{ \pm}\right)$satisfies the first two conditions for an EZ-structure. To verify the remaining properties, we first observe that an easy adaptation of an argument of Bestvina (Proposition 1.18 in [4]) shows that in $\mathbb{D}^{m}$, there exists a neighborhood base $\left\{V_{i}\right\}$ of the point $S^{+}$which has the following properties:

(1) $V_{i+1} \subset V_{i}$ for every $i$,

(2) for every compact set $K \subset \mathbb{D}^{m}-\left\{S^{ \pm}\right\}$, there exists a $k$ such that $g^{k}(K) \subset V_{1}$,

(3) there exists a fixed $j$ such that $g^{j}\left(V_{i}\right)=V_{i+1}$ for every $i$.

Here $g$ denotes the generator of $S$ whose positive powers tend to $S^{+}$.

We now explain how proper discontinuity of the action follows. Note that, by hyperbolicity of the $\Gamma$-action on $\partial^{\infty} \Gamma, S$ restricted to $\partial^{\infty} \Gamma-\left\{S^{ \pm}\right\}$acts properly discontinuously. Hence if proper discontinuity fails at $p$, then $p$ is an element of $\partial^{\infty} \Gamma$, and one can construct sequences $x_{i} \in \mathbb{D}^{m}-\partial^{\infty} \Gamma$ and $n_{i} \in \mathbb{Z}$ such that $x_{i} \rightarrow S^{+}, n_{i} \rightarrow+\infty$, and $g^{n_{i}}\left(x_{i}\right) \rightarrow p$. But this immediately contradicts the existence of the family $\left\{V_{i}\right\}$ given above. Hence the action of $S$ on $\mathbb{D}^{m}-\left\{S^{ \pm}\right\}$is properly discontinuous. Then the freeness of the $S$-action is also immediate, since the $\Gamma$ action (and hence the $S$-action) on $\mathbb{D}^{m}-\partial^{\infty} \Gamma$ is free, while the $S$-action on $\partial^{\infty} \Gamma$ fixes precisely the two points $S^{ \pm}$. The null-sequence property follows from the fact that the $S$-action is properly discontinuous on $\mathbb{D}^{m}-\left\{S^{ \pm}\right\}$, and the fact that $\mathbb{D}^{m}$ is the 2-point compactification of $\mathbb{D}^{m}-\left\{S^{ \pm}\right\}$.

Finally, to see cocompactness, identify $\mathbb{D}^{m}-\left\{S^{ \pm}\right\}$with $\mathbb{D}^{m-1} \times \mathbb{R}$ so that $S^{+}$ corresponds to $+\infty$. Since the $S$-action is properly discontinuous, there exists an integer $n>0$ such that $g^{n}\left(\mathbb{D}^{m-1} \times\{0\}\right) \subset \mathbb{D}^{m-1} \times(0,+\infty)$. Let $W$ be the region between $\mathbb{D}^{m-1} \times\{0\}$ and $g^{n}\left(\mathbb{D}^{m-1} \times\{0\}\right)$; i.e.

$$
W=g^{n}\left(\mathbb{D}^{m-1} \times(-\infty, 0]\right) \cap \mathbb{D}^{m-1} \times[0, \infty) .
$$


$W$ is clearly compact, and it is not difficult to see that $\cup_{i \in \mathbb{Z}}\left[g^{i n}(W)\right]=\mathbb{D}^{m-1} \times \mathbb{R}$, which establishes that $S$ acts cocompactly on $\mathbb{D}^{m}-\left\{S^{ \pm}\right\}$.

We now have that the pair $\left(\mathbb{D}^{m}, S^{ \pm}\right)$satisfies all the conditions for an EZ-structure, concluding the proof of Claim 1.

We now continue the proof of Theorem 1.3. Note that

$$
\left(\mathbb{D}^{m+1},\left\{S^{ \pm}\right\}\right)=\left(\mathbb{D}^{m},\left\{S^{ \pm}\right\}\right) \times I .
$$

Arguing as in the paper by Farrell-Jones (see pgs. 462-467 in [10]), it suffices to construct, for each sufficiently large integer $m$, a pair of continuous maps:

$$
\begin{aligned}
& g_{S}: P\left(M_{S}^{m}\right) \longrightarrow P\left(M^{m}\right) \\
& g^{S}: P\left(M^{m}\right) \longrightarrow P\left(M_{S}^{m}\right)
\end{aligned}
$$

where $M^{m}=\left(\mathbb{D}^{m}-\partial^{\infty} \Gamma\right) / \Gamma, M_{S}^{m}=\left(\mathbb{D}^{m}-\left\{S^{ \pm}\right\}\right) / S$, and $P(\cdot)$ denotes the (unstable) topological pseudo-isotopy space, and where the maps $g_{S}$ and $g^{S}$ satisfy the following:

Assertion. $g^{S} \circ g_{S}$ is homotopic to the identity, and $g^{S^{\prime}} \circ g_{S}$ is homotopic to a constant map whenever $S \neq S^{\prime}$.

We first discuss the construction of the maps $g_{S}, g^{S}$, and will then discuss why the pair of maps we constructed satisfy the assertion. Start by observing that both $M^{m}$ and $M_{S}^{m}$ are compact $m$-dimensional manifolds with boundary (we will henceforth suppress the superscript indicating dimension unless it is explicitly relevant to the argument being presented). Now let $p=p_{S}: \operatorname{Int}\left(M_{S}\right) \rightarrow \operatorname{Int}(M)$ be the covering space corresponding to the subgroup $S \subset \Gamma=\pi_{1}(\operatorname{Int}(M))$. Using the s-cobordism theorem (and assuming $m \geq 6$ ), one easily constructs an isotopy $\phi_{t}=\phi_{t}^{S}: M_{S} \rightarrow M_{S}$ such that $\phi_{0}=\operatorname{Id}_{M_{S}}$, and $p \circ \phi_{1}: M_{S} \rightarrow M$ is an embedding. To define $g_{S}$, let $f: M_{S} \times I \rightarrow M_{S} \times I$ be a pseudo-isotopy (i.e. an element of $P\left(M_{S}\right)$ ). Recall that $f$ is an automorphism (i.e. an onto homeomorphism) with the property that:

$$
\left.f\right|_{M_{S} \times\{0\} \cup\left(\partial M_{S}\right) \times I}=\left.\mathrm{Id}\right|_{M_{S} \times\{0\} \cup\left(\partial M_{S}\right) \times I} .
$$

We can now define $f_{*}=g_{S}(f) \in P(M)$ by setting $f_{*}(x, t)$ to be:

- $(x, t)$ if $x \in M-\operatorname{Image}\left(p \circ \phi_{1}\right)$

- $p \circ \phi_{1}(f(\bar{x}, t))$ if $x=p \circ \phi_{1}(\bar{x})$

where $x \in M$ and $t \in I$. This gives us the map $g_{S}$.

On the other hand, to define $g^{S}(f)$, where $f \in P(M)$, let $\tilde{f}:\left(\mathbb{D}^{m}-\partial^{\infty} \Gamma\right) \times I \rightarrow$ $\left(\mathbb{D}^{m}-\partial^{\infty} \Gamma\right) \times I$ be the lift of $f$ such that $\tilde{f}(x, t)=(x, t)$ if either $x \in S^{m-1}=$ $\partial \mathbb{D}^{m}$ or if $t=0$. Now $\tilde{f}$ induces an automorphism $\bar{f}$ of $\left(\mathbb{D}^{m+1}, \partial^{\infty} \Gamma\right)$, since $\left(\mathbb{D}^{m+1}, \partial^{\infty} \Gamma\right)=\left(\mathbb{D}^{m}, \partial^{\infty} \Gamma\right) \times I$. Note that $\bar{f}$ is $\Gamma$-equivariant and that $\left.\bar{f}\right|_{\partial_{-} \mathbb{D}^{m+1}}=$ 
$\mathrm{Id}_{\partial_{-}-\mathbb{D}^{m+1}}$, where $\partial_{-} \mathbb{D}^{m+1}$ is the image of $\mathbb{D}^{m} \times\{0\} \cup S^{m-1} \times I$ under the quotient map $\mathbb{D}^{m} \times I \rightarrow \mathbb{D}^{m+1}$. Since $\partial^{\infty} \Gamma \subset \partial_{-} \mathbb{D}^{m+1}, \bar{f}$ induces an $S$-equivariant automorphism of $\mathbb{D}^{m+1}-\left\{S^{ \pm}\right\}$which then descends to an automorphism $f_{S}$ of $\left(\mathbb{D}^{m+1}-\left\{S^{ \pm}\right\}\right) / S$. After "appropriately identifying"

$$
\mathcal{M}_{S}=\left(\mathbb{D}^{m+1}-\left\{S^{ \pm}\right\}\right) / S
$$

with $M_{S}^{m} \times I, g^{S}(f)$ is defined by $g^{S}(f)=f_{S}$.

To do this identification, first note that $\mathcal{M}_{S}$ is the quotient space of $M_{S}^{m} \times I$ where each interval $x \times I, x \in \partial M_{S}^{m}$ is collapsed to a point. So $M_{S}^{m} \times\{0\}$ is canonically identified with a codimension zero submanifold $\partial_{-} \mathcal{M}_{S}$ of $\partial \mathcal{M}_{S}$. By equating $\partial M_{S}^{m} \times I$ with a short collar of $\partial\left(\partial_{-} \mathcal{M}_{S}\right)$ in $\partial_{-} \mathcal{M}_{S}$, an identification of $M_{S} \times I$ to $\mathcal{M}_{S}$ can be constructed such that the composition

$$
P\left(M_{S}\right) \longrightarrow \operatorname{Aut}\left(\mathcal{M}_{S}, \partial_{-}\left(\mathcal{M}_{S}\right)\right) \longrightarrow P\left(M_{S}\right)
$$

is homotopic to the identity (here the two maps above are the naturally defined continuous maps; in fact, the second map is the homeomorphism induced by the identification while the first is determined by the fact that $\mathcal{M}_{S}$ is a quotient space of $M_{S} \times I$ ). This is the "appropriate identification" mentioned above.

This gives us the two maps for which we claim the assertion holds. Before continuing our proof, we note that, when $m \geq 6$, the spaces $M_{S}^{m}$ are all homeomorphic to $S^{1} \times \mathbb{D}^{m-1}$. Indeed, this follows by the s-cobordism theorem, and the fact that $S$ acts via orientation preserving homeomorphisms on $\mathbb{D}^{m}-\left\{S^{ \pm}\right\}$; thus the closed tubular neighborhood of any embedded circle $S^{1}$ in $\operatorname{Int}\left(M_{S}^{m}\right)$, which induces a homotopy equivalence, is homeomorphic to $S^{1} \times \mathbb{D}^{m-1}$.

Now the Assertion, made above, can be verified in the same way that properties (i) and (ii) in Lemma 2.1 of Farrell-Jones [10] were proven. We merely point out that they follow directly from the following two claims which we proceed to formulate and then to verify. Let $T_{S}$ denote the image of $p_{S} \circ \phi_{1}^{S}$. Note that $T_{S}$ is a codimension zero submanifold of $\operatorname{Int}\left(M_{S}^{m}\right)$ and that $T_{S}$ is homeomorphic to $S^{1} \times \mathbb{D}^{m-1}$. Recall that

$$
p_{S}: \operatorname{Int}\left(M_{S}\right) \longrightarrow \operatorname{Int}(M)
$$

is the covering projection corresponding to $S \subset \Gamma$. And that $\phi_{1}^{S}: M_{S} \rightarrow \operatorname{Int}\left(M_{S}\right)$ is an embedding isotopic to $\operatorname{Id}_{M_{S}}$. Recall that we assumed that $\Gamma$ is not cyclic.

Now let $\left\{C_{i}\right\}$ denote the connected components of $p_{S}^{-1}\left(T_{S}\right)$, and note that $p_{S}^{-1}=$ $\bigsqcup_{i} C_{i}$. Let $\bar{C}_{i}$ denote the closure of $C_{i}$ in $M_{S}$. It is an elementary observation that each $C_{i}$ is a codimension zero submanifold of $\operatorname{Int}\left(M_{S}\right)$ as well as an open subset of $p_{S}^{-1}\left(T_{S}\right)$. Furthermore, observe that $\operatorname{Image}\left(\phi_{1}^{S}\right)$ is a codimension zero submanifold of $\operatorname{Int}\left(M_{S}\right)$ which is homeomorphic to $S^{1} \times \mathbb{D}^{m+1}$.

Claim 2. We can index the set $\left\{C_{i}\right\}$ so that $C_{0}=\operatorname{Image}\left(\phi_{S}^{1}\right)$ and $\bar{C}_{i}$ is homeomorphic to $\mathbb{D}^{m}$ when $i \neq 0$. 
Now let $S^{\prime} \in \mathcal{M}$ with $S^{\prime} \neq S$, and denote by $\left\{C_{i}^{\prime}\right\}$ the connected components of $p_{S^{\prime}}^{-1}\left(T_{S}\right)$ and by $\bar{C}_{i}^{\prime}$ the closure of $C_{i}^{\prime}$ in $M_{S^{\prime}}$. It is again elementary that each $C_{i}^{\prime}$ is a codimension zero submanifold of $\operatorname{Int}\left(M_{S^{\prime}}\right)$ as well as an open subset of $p_{S^{\prime}}^{-1}\left(T_{S}\right)$.

Claim 3. Each $\bar{C}_{i}^{\prime}$ is homeomorphic to $\mathbb{D}^{m}$.

We now proceed with the proofs of the two claims. The Facts $1_{\delta}-4_{\delta}$ used in the proofs below refer to the facts about $\delta$-hyperbolic groups discussed at the beginning of this section.

Proof of Claim 2. One easily sees that each $p_{i}: C_{i} \rightarrow T_{S}$ is a covering projection where $p_{i}=\left.p_{S}\right|_{C_{i}}$. Hence Image $\left(\phi_{1}^{S}\right)$ must be one of the components $C_{i}$ since $p:$ Image $\left(\phi_{i}^{S}\right) \rightarrow T_{S}$ is a homeomorphism. Thus we may index the components starting with $C_{0}=\operatorname{Image}\left(\phi_{1}^{S}\right)$. Therefore it remains to show that $\bar{C}_{i}$ is homeomorphic to $\mathbb{D}^{m}$ when $i \neq 0$. To do this, define

- $q: \mathbb{D}^{m}-\partial^{\infty} \Gamma \longrightarrow M=\left(\mathbb{D}^{m}-\partial^{\infty} \Gamma\right) / \Gamma$

- $r=r_{S}: \mathbb{D}^{m}-\left\{S^{ \pm}\right\} \longrightarrow M_{S}=\left(\mathbb{D}^{m}-\left\{S^{ \pm}\right\}\right) / S$

to be the universal covering maps whose groups of deck transformations are $\Gamma$ and $S$ respectively. Then we have the following commutative triangle of covering spaces:

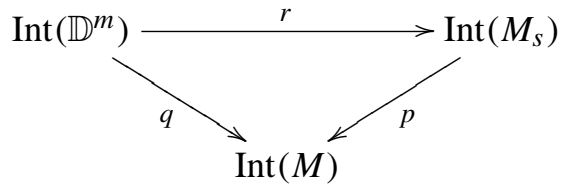

Note that $q^{-1}\left(T_{S}\right)=\bigsqcup_{i} D_{i}$ where each $D_{i}$ is a connected component of $q^{-1}\left(T_{S}\right)$. And let $\bar{D}_{i}$ be the closure of $D_{i}$ in $\mathbb{D}^{m}$. One easily sees the following ten points:

(1) Each $D_{i}$ is open in $q^{-1}\left(T_{S}\right)$.

(2) Each $D_{i}$ is a codimension zero submanifold of $\operatorname{Int}\left(\mathbb{D}^{m}\right)$.

(3) $q_{i}: D_{i} \rightarrow T_{S}$ is a universal covering space (where $q_{i}=\left.q\right|_{D_{i}}$ ) whose group of deck transformations $S_{i}$ consists of all $\gamma \in \Gamma$ such that $\gamma\left(D_{i}\right)=D_{i}$. Consequently, $D_{i}$ is homeomorphic to $\mathbb{D}^{m-1} \times \mathbb{R}$.

(4) The components $D_{i}$ are permuted transitively by $\Gamma$. Consequently, the groups $S_{i}$ are all conjugate cyclic subgroups of $\Gamma$.

(5) At least one of the groups $S_{i}$ is $S$. Hence all the $S_{i}$ are maximal cyclic subgroups of $\Gamma$. And we can rearrange the indexing so that $S_{0}=S$.

(6) If the cardinality $\left|S_{i} \cap S_{j}\right|>1$, then $i=j$. This follows from points (4) and (5) by using Fact $1_{\delta}$ and Fact $2_{\delta}$.

(7) Let $\tilde{\phi}_{t}: \mathbb{D}^{m}-\left\{S^{ \pm}\right\} \rightarrow \mathbb{D}^{m}-\left\{S^{ \pm}\right\}$be the lift of the isotopy $\phi_{t}$ with respect to the covering projection $r$ such that $\tilde{\phi}_{0}=$ Id. Then $D_{0}=\operatorname{Image}\left(\tilde{\phi}_{1}\right)$, and consequently $\bar{D}_{0}=D_{0} \cup\left\{S^{ \pm}\right\}$, which forces $\bar{D}_{0}$ to be homeomorphic to $\mathbb{D}^{m}$. 
(8) Because of points (4) and (7), $\bar{D}_{i}=D_{i} \cup\left\{S_{i}^{ \pm}\right\}$and is homeomorphic to $\mathbb{D}^{m}$. Also because of point (6) and Fact $3_{\delta}, \bar{D}_{i} \subset \mathbb{D}^{m}-\left\{S^{ \pm}\right\}$if $i \neq 0$, and consequently $\bar{D}_{i}$ is also the closure of $D_{i}$ in $\mathbb{D}^{m}-\left\{S^{ \pm}\right\}$.

(9) If $\gamma\left(\bar{D}_{i}\right) \cap \bar{D}_{i} \neq \emptyset$, where $\gamma \in \Gamma$, then $\gamma \in S_{i}$. This results from points (4), (6), (8), along with Facts $3_{\delta}$ and $4_{\delta}$. Consequently, if $i \neq 0$, then $\left.r\right|_{\bar{D}_{i}}: \bar{D}_{i} \rightarrow$ $r\left(\bar{D}_{i}\right)=\overline{r\left(D_{i}\right)}$ is a homeomorphism since $S_{i} \cap S_{0}=1$, because of point (6) (Here $\overline{r\left(D_{i}\right)}$ denotes the closure of $r\left(D_{i}\right)$ in $M_{S}$ ).

(10) There is a surjection of indexing sets $i \mapsto \alpha(i)$, with $\alpha(0)=0$, such that $r_{i}: D_{i} \rightarrow C_{\alpha(i)}$ is a covering space (here $r_{i}$ denotes $\left.r\right|_{D_{i}}$ ). This follows from the above commutative triangle in which $p, q$, and $r$ are open maps.

It now follows immediately from points (8), (9), and (10), that $\bar{C}_{i}$ is homeomorphic to $\mathbb{D}^{m}$ when $i \neq 0$; thus completing the proof of Claim 2 .

Proof of Claim 3. This proof closely parallels the one just given for Claim 2. Note that the above points (1)-(9) continue to hold. And by replacing $S$ by $S^{\prime}$ in the above commutative triangle, the following analogue (10)' of point (10) is similarly verified using that $p_{S^{\prime}}, q$, and $r_{S^{\prime}}$ are open maps: there is a surjection $i \mapsto \beta(i)$ of indexing sets such that $r_{i}^{\prime}: D_{i} \rightarrow C_{\beta(i)}^{\prime}$ is a covering space where $r_{i}^{\prime}=\left.r_{S^{\prime}}\right|_{D_{i}}$.

Then Fact $3_{\delta}$ yields that:

$$
\left\{S_{i}^{ \pm}\right\} \subseteq\left(\mathbb{D}^{m}-\partial^{\infty} S^{\prime}\right)=\operatorname{Domain}\left(r_{S^{\prime}}\right)
$$

which together with point (8) shows that

$$
\bar{D}_{i} \subseteq \operatorname{Domain}\left(r_{S^{\prime}}\right)
$$

Therefore point (9) yields that:

$$
\left.r_{S^{\prime}}\right|_{\bar{D}_{i}}: \bar{D}_{i} \longrightarrow r_{S^{\prime}}\left(\bar{D}_{i}\right)=\overline{r_{S^{\prime}}\left(D_{i}\right)}=\overline{C_{\beta(i)}^{\prime}}
$$

is a homeomorphism. But $\bar{D}_{i}$ is homeomorphic to $\mathbb{D}^{m}$ by point (8), and $\beta$ is a surjection by point $(10)^{\prime}$. This concludes the proof of Claim 3 .

Finally, we point out that, from these two claims, it is easy to show the Assertion. Indeed, the pseudo-isotopies $g^{S} \circ g_{S}(f)$ and $g^{S^{\prime}} \circ g_{S}(f)$ are supported over $\bigcup_{i} \bar{C}_{i}$ and $\bigcup_{i} \bar{C}_{i}^{\prime}$ respectively. Because of claims 2 and 3 , the Alexander trick can be used to verify the Assertion. We refer the reader to Section 2 of Farrell-Jones [10] for more details. 


\section{References}

[1] Ancel, F. D., and Guilbault, C. R., Z-compactifications of open manifolds. Topology 38 (1999), no. 6, 1265-1280. Zbl 0951.57011 MR 1690157

[2] Benoist, Y., Foulon, P., and Labourie, F., Flots d'Anosov à distributions stable et instable différentiables. (French) [Anosov flows with stable and unstable differentiable distributions]. J. Amer. Math. Soc. 5 (1992), no. 1, 33-74. Zbl 0759.58035 MR 1124979

[3] Bestvina, M., and Mess, G., The boundary of negatively curved groups. J. Amer. Math. Soc. 4 (1991), no. 3, 469-481. Zbl 0767.20014 MR 1096169

[4] Bestvina, M., Local homology properties of boundaries of groups. Michigan Math. J. 43 (1996), no. 1, 123-139. Zbl 0872.57005 MR 1381603

[5] Bridson, M., and Haefliger, A., Metric spaces of non-positive curvature. Grundlehren Math. Wiss. 319, Springer-Verlag, Berlin, 1999. Zbl 0988.53001 MR 1744486

[6] Carlsson, G., and Pedersen, Erik K., Controlled algebra and the Novikov conjectures for $K$ - and $L$-theory. Topology 34 (1995), no. 3, 731-758. Zbl 0838.55004 MR 1341817

[7] Edwards, R. D., The topology of manifolds and cell-like maps. In Proceedings of the International Congress of Mathematicians (Helsinki, 1978), Acad. Sci. Fennica, Helsinki, 1980, 111-127. Zbl 0428.57004 MR 0562601

[8] Farrell, F. T., and Hsiang, W. C., On Novikov's conjecture for nonpositively curved manifolds. I. Ann. of Math. (2) 113 (1981), no. 1, 199-209. Zbl 0461.57016 MR 0604047

[9] Farrell, F. T., and Hsiang, W. C., On Novikov's conjecture for cocompact discrete subgroups of a Lie group. In Algebraic topology, Aarhus 1982, Lecture Notes in Math. 1051, SpringerVerlag, Berlin, 1984, 38-48. Zbl 0543.57014 MR 0764575

[10] Farrell, F. T., and Jones, L. E., K-theory and dynamics. II. Ann. of Math. (2) 126 (1987), no. 3, 451-493. Zbl 0665.58038 MR 0916716

[11] Farrell, F. T., and Jones, L. E., Isomorphism conjectures in algebraic $K$-theory. J. Amer. Math. Soc. 6 (1993), no. 2, 249-297. Zbl 0798.57018 MR 1179537

[12] Farrell, F. T., Lectures on surgical methods in rigidity. Published for the Tata Institute of Fundamental Research, Bombay, by Springer-Verlag, Berlin, 1996. Zbl 0883.57035 MR 1452860

[13] Farrell, F. T., The Borel conjecture. In Topology of high-dimensional manifolds, Vol. 1 (eds. F. T. Farrell, L. Goettsche and W. Lueck), ICTP Lecture Notes 9, Trieste, 2002, 225-298. Zbl 1042.57001 MR 1937017

[14] Ferry, S. C., and Weinberger, S., Curvature, tangentiality, and controlled topology. Invent. Math. 105 (1991), no. 2, 401-414. Zbl 0744.57017 MR 1115548

[15] Hatcher, A. E., Concordance spaces, higher simple-homotopy theory, and applications. In Algebraic and geometric topology (Proc. Sympos. Pure Math., Stanford Univ., Stanford, Calif., 1976), Part 1, Proc. Sympos. Pure Math. XXXII, Amer. Math. Soc., Providence, RI, 1978, 3-21. Zbl 0406.57031 MR 0520490

[16] Hu, B., Retractions of closed manifolds with nonpositive curvature. In Geometric group theory (Columbus, OH, 1992), Ohio State Univ. Math. Res. Inst. Publ. 3, de Gruyter, Berlin, 1995, 135-147. Zbl 0842.57003 MR 1355108 
Vol. 80 (2005)

[17] Igusa, K., What happens to Hatcher and Wagoner's formulas for $\pi_{0} C(M)$ when the first Postnikov invariant of $M$ is nontrivial? In Algebraic $K$-theory, number theory, geometry and analysis (Bielefeld, 1982), Lecture Notes in Math. 1046, Springer-Verlag, Berlin, 1984, 104-172. Zbl 0546.57015 MR 0750679

[18] Kirby, R. C., and Siebenmann, L. C., Foundational essays on topological manifolds, smoothings, and triangulations. Ann. of Math. Stud. 88, Princeton University Press, Princeton, NJ, 1977. Zbl 0361.57004 MR 0645390

[19] Milnor, J., Lectures on the h-cobordism theorem. Princeton University Press, Princeton, NJ, 1965. Zbl 0161.20302 MR 0190942

[20] Mio, W., Homology manifolds. In Surveys on surgery theory, Vol. 1, Ann. of Math. Stud. 145, Princeton University Press, Princeton, NJ, 2000, 323-343. Zbl 0946.57002 MR 1747540

[21] Quinn, F., Ends of maps, I. Ann. of Math. (2) 110 (1979), 275-331. Zbl 394.57022 MR 0549490

[22] Quinn, F., Resolutions of homology manifolds, and the topological characterization of manifolds. Invent. Math. 72 (1983), no. 2, 267-284. Zbl 0555.57003 MR 0700771

[23] Quinn, F., An obstruction to the resolution of homology manifolds. Michigan Math. J. 34 (1987), no. 2, 285-291. Zbl 0652.57011 MR 0894878

[24] West, J. E., Mapping Hilbert cube manifolds to ANR's: a solution of a conjecture of Borsuk. Ann. of Math. (2) 106 (1977), no. 1, 1-18. Zbl 0375.57013 MR 0451247

Received June 3, 2003

F. T. Farrell, J.-F. Lafont, Department of Mathematical Sciences, S.U.N.Y. Binghamton, Binghamton, NY 13902-6000, U.S.A.

E-mail: farrell@math.binghamton.edu; jlafont@math.binghamton.edu 